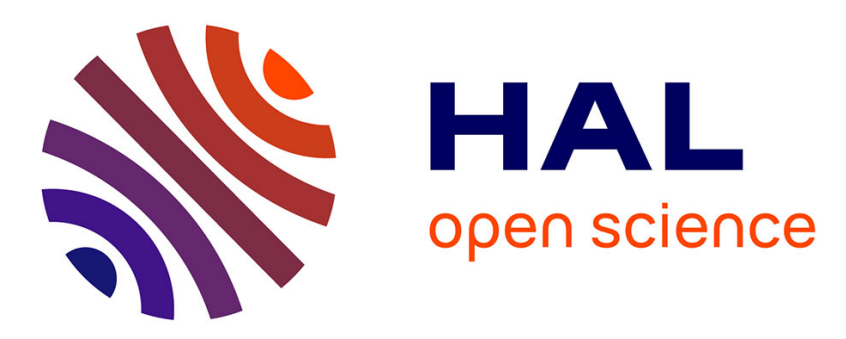

\title{
A quaternion-based Complementary Sliding Mode Observer for attitude estimation: application in free-ranging animal motions
}

Hassen Fourati, Noureddine Manamanni, Lissan Afilal, Yves Handrich

\section{- To cite this version:}

Hassen Fourati, Noureddine Manamanni, Lissan Afilal, Yves Handrich. A quaternion-based Complementary Sliding Mode Observer for attitude estimation: application in free-ranging animal motions. 49th IEEE Conference on Decision and Control, Dec 2010, Atlanta, United States. pp. 5056-5061. hal-00642928

\section{HAL Id: hal-00642928 \\ https://hal.science/hal-00642928}

Submitted on 19 Nov 2011

HAL is a multi-disciplinary open access archive for the deposit and dissemination of scientific research documents, whether they are published or not. The documents may come from teaching and research institutions in France or abroad, or from public or private research centers.
L'archive ouverte pluridisciplinaire HAL, est destinée au dépôt et à la diffusion de documents scientifiques de niveau recherche, publiés ou non, émanant des établissements d'enseignement et de recherche français ou étrangers, des laboratoires publics ou privés. 


\title{
A quaternion-based Complementary Sliding Mode Observer for attitude estimation: application in free-ranging animal motions
}

\author{
H. Fourati ${ }^{1,2}$, Student, IEEE, N. Manamanni ${ }^{1}$,Member, IEEE, A. B. Jemaa ${ }^{1}$, L. Afilal ${ }^{1}$ \\ and Y. Handrich ${ }^{2}$
}

\begin{abstract}
This paper proposes a sensing method using a module of GAM sensors (3-axis Gyroscope, 3-axis Accelerometer, 3-axis Magnetometer) for more accurate measurement of the marine animal's posture. The application in mind is that of Bio-logging. A quaternion-based algorithm is developed through the complementary filtering theory in order to combine all information sources and to obtain an accurate estimation of the attitude. By considering the rigid body kinematic model, a Complementary Sliding Mode Observer (CSMO) is proposed, taking advantages from strapdown inertial measurements and vector observations. The proposed algorithm is based on the multiplicative quaternion correction technique. The performance of the CSMO is shown with a set of preliminary experiments on domestic animals through sensor measurements provided by an Inertial Measurement Unit.
\end{abstract}

\section{INTRODUCTION}

Nowadays, the attitude estimation of a rigid body has attracted a strong interest in many fields such as Walking Robots [1], Micro Air Vehicles [2], Marine Vehicles [3] and others. The need of reliable and precise attitude information is one of the fundamental requirements in these areas. Note that, the progress in Micro Electro-Mechanical System (MEMS) has enabled the development of low-cost inertial and magnetic sensors, namely, the gyroscopes, the accelerometers, and the magnetometers. Therefore, the use of these sensors in the attitude estimation problem has attracted a strong interest.

The attitude determination process usually involves some errors (e.g., sensor noise, bias, and inaccuracy in predictions). For this reason, most of the attitude determination methods aim to combine two or more sensor measurements in an optimal way and use state estimation techniques to increase the accuracy of the solutions. Quaternion has been the subject of studies in many attitude determination systems using various filtering theories. Due to the unconventional nature of quaternion kinematics, filter models have been synthesized in two different ways related to the objectives, the formulation of the measurement error vector and the update of the state estimates. The first way is based on additive quaternion correction [2]-[6]. This approach is easy to implement but it is considered as

Manuscript received March 30, 2010. This work was supported by Alsace and Champagne-Ardenne Regions within the framework of the project "NaviMeles".

${ }^{1}$ H. Fourati, A. Ben Jemaa, N. Manamanni and L. Afilal are with CReSTIC-URCA, Reims Champagne Ardenne University, France noureddine.manamanni@univ-reims.fr

${ }^{2}$ Y. Handrich and H. Fourati are with IPHC-DEPE-CNRS, Strasbourg University, France. yves-jean.handrich@c-strasbourg.fr localized approximation since it is valid only for small attitude changes. The second way uses multiplicative quaternion correction [7]-[9] and can be applied for larger attitude maneuvers.

This paper focuses on the development of a robust method recovering the full attitude of a moving rigid body and using the multiplicative quaternion correction. The application in mind is that of Bio-logging. It is one of the recent interesting areas, at the intersection of the animal behavior and bioengineering, where the attitude estimation is becoming a requirement for biologists. It aims to obtain new information on the natural world and provides new insights into the hidden lives of animal species [10]. Bio-logging generally involves attaching an electronic device (biologger) to an animal in order to record different aspects of its biology and environment. During the last 10 years, a considerable number of studies have been undertaken to estimate the posture or orientation of marine mammals using only accelerometers and magnetometers [11]-[13]. However, these works are not well suited in dynamic situations observed underwater for marine animals, e.g. the phases of prey pursuit. Therefore, the estimation performance is significantly degraded.

This paper proposes an alternative approach to estimate the attitude in free-ranging marine animals during their movements to overcome the drawbacks of mentioned papers in this area [11]-[13]. The main idea is to use a Complementary Sliding Mode Observer (CSMO) that exploits the multiplicative quaternion correction technique to recover the full rigid body orientation. The nonlinear observer is fed with inertial and magnetic measurements and takes into account the complementary spectra of these signals. In fact, the estimation algorithm idea uses 3-axis gyroscope measurements to derive the attitude. The correction was performed using a quaternion error continuously derived from a 3 -axis accelerometer and 3-axis magnetometer data fusion method. This reduces the integration drift that originates from the angular velocity.

This paper is organized as follows: Section II gives some backgrounds of the rigid body attitude representation. The formulation of the CSMO is proposed in section III. Section IV is devoted to preliminary experimental results to illustrate the efficiency of the proposed approach. Section V summarizes the main conclusions of the paper.

\section{RIGID BODY ATTITUDE DESCRIPTION}

As known the rigid body attitude is the orientation relation between the body frame $B\left(X_{B}, Y_{B}, Z_{B}\right)$ and local 
navigation frame $N\left(X_{N}, Y_{N}, Z_{N}\right)$ (see Fig. 1). The former is fixed to the rigid-body. The latter is tangent to the Earth's surface (Local Tangent Plane, LTP) and it is particularly useful to express the attitude of a moving rigid body on the surface of the earth [14]. The $X_{N}$-axis points towards the true north. The $Z_{N}$-axis points towards the interior of the Earth, perpendicular to the reference ellipsoid. The $Y_{N}$-axis completes the right-handed coordinate system, pointing east (NED: North, East, Down).

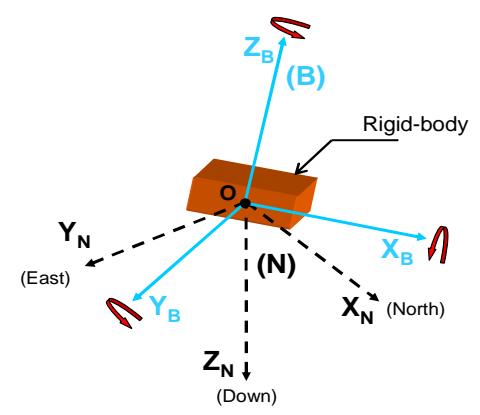

Fig. 1. The coordinate system (B) (solid line) of a rigid body represented in the Earth-fixed frame $(\mathrm{N})$ (dashed line)

Recall that the unit quaternion is the mathematical representation of the rigid body attitude between the frames $B$ and $N$. The quaternion, denoted by $q$, is a hypercomplex number of rank 4 such that:

$$
q=q_{0}+q_{1} i+q_{2} j+q_{3} k=\left[\begin{array}{ll}
\cos \frac{\phi}{2} & \vec{u} \sin \frac{\phi}{2}
\end{array}\right]^{T}=\left[\begin{array}{c}
q_{0} \\
q_{\text {vect }}
\end{array}\right]
$$

where $q_{0}, q_{1}, q_{2}$ and $q_{3}$ are real numbers. $i, j$, and $k$ are the components of the vector $\vec{u}$ (Euler axis), $\phi$ is the rotation angle and $q_{\text {vect }}=\left[\begin{array}{lll}q_{1} & q_{2} & q_{3}\end{array}\right]^{T} \in \Re^{3 \times 1}$ is the imaginary vector. More details about quaternion algebra can be founded in [15].

\section{COMPLEMENTARY SLIDING MODE OBSERVER DESIGN}

In this section, the objective is to design a rigid body attitude estimation algorithm based on inertial and magnetic MEMS sensors. By considering the rigid body kinematic model, a CSMO is proposed in order to take advantage from the good short term precision given by rate gyros integration and the reliable long term accuracy provided by accelerometers and magnetometers measurements. Hence, the CSMO exploits the low-frequency region where the attitude observations are typically more accurate, and the high-frequency region where the integration of the rate gyro yields better attitude estimates. It is important to note that the resulting structure is complementary: high bandwidth rate gyro measurements are combined with low bandwidth vector observations.

\section{A. Rigid body kinematic model}

The attitude kinematic equation describes the time rate of change of the attitude $q$, in a quaternion term, as a result of the rigid body angular rates measured by the gyroscope. Therefore, the quaternion derivative that represents the kinematic differential equation is given as in [16] as follows:

$$
\frac{\partial q}{\partial t}=\frac{1}{2} \bar{\omega}_{g} \otimes q
$$

where $\bar{\omega}_{g}=\left[\begin{array}{ll}0 & \omega_{g}\end{array}\right]^{T}$ is the quaternion form of the angular velocity vector $\omega_{g}=\left[\begin{array}{lll}\omega_{g x} & \omega_{g y} & \omega_{g z}\end{array}\right]^{T}$.

The angular velocities measurements $\omega_{g}$ are often corrupted with noises and bias $w(t)$ [17]. Therefore, including gyroscope disturbances and using the quaternion product $\otimes$ given in [15], (2) can be written such as:

$$
\frac{\partial q}{\partial t}=\frac{1}{2}\left[\begin{array}{c}
-q_{\text {vect }}^{T} \\
I_{3 \times 3} q_{0}+\left[q_{\text {vect }}^{\times}\right]
\end{array}\right] \omega_{g}+w(t)
$$

where $\left[q_{v e c t}^{\times}\right]$represents the skew-symmetric matrix defined as [2]:

$$
\left[q_{\text {vect }}^{\times}\right]=\left[\begin{array}{c}
q_{1} \\
q_{2} \\
q_{3}
\end{array}\right]^{\times}=\left[\begin{array}{ccc}
0 & -q_{3} & q_{2} \\
q_{3} & 0 & -q_{1} \\
-q_{2} & q_{1} & 0
\end{array}\right]
$$

and $I_{3 \times 3}$ is the identity matrix of dimension 3 .

Hence, let us consider the following nonlinear system composed of (3) and the linear measurement model $y$ :

$$
\left\{\begin{array}{l}
\frac{\partial q}{\partial t}=\frac{1}{2}\left[\begin{array}{c}
-q_{v e c t}^{T} \\
I_{3 \times 3} q_{0}+\left[q_{v e c t}^{\times}\right]
\end{array}\right] \omega_{g}+w(t) \\
y=C q+v
\end{array}\right.
$$

where $q=\left[\begin{array}{llll}q_{0} & q_{1} & q_{2} & q_{3}\end{array}\right]^{T}$ represents the quaternion vector. The linear measurement model $y$ is taken as follows $y=q_{m} \in \mathfrak{R}^{4 \times 1}$ and $C=I_{4 \times 4} . v$ represents the vector of the measured noises. It is important to note that the rigid body quaternion measurements $q_{m}$ may be determined from accelerometer and magnetometer readings.

\section{B. Quaternion $q_{m}$ calculation from accelerometer and magnetometer measurements}

The problem of optimal attitude determination algorithm using vector observations is known in the literature as Wahba's problem [18], [19]. The magnetometer provides the magnetic field vector in the frame $B$. This quantity is locally constant in the frame $N$ and therefore it represents a vector observation. For sufficiently low frequency bandwidths, the gravitational field also dominates the accelerometer measurements, as discussed in [2]. This would provide a second vector observation.

For this purpose, a Levenberg Marquardt Algorithm (LMA) is proposed to recover complete posture information, expressed in quaternion term, by using a 3-axis accelerometer and a 3-axis magnetometer [9]. 
The 3-axis accelerometer measures the specific force $f$ in the body frame $B$ as follows:

$$
f=\left[\begin{array}{lll}
f_{x} & f_{y} & f_{z}
\end{array}\right]^{T}=M_{N}^{B}(q)[a+g]+\delta_{f}
$$

where $g=\left[\begin{array}{lll}0 & 0 & 9.81\end{array}\right]^{T}$ and $a \in \mathfrak{R}^{3}$ represent respectively the gravity vector and the inertial acceleration of the body, expressed in the navigation frame $N . \delta_{f} \in \mathfrak{R}^{3}$ is the vector of zero-mean white Gaussian noise. The rotation matrix $M_{N}^{B}(q)$ is expressed as:

$$
M_{N}^{B}(q)=\left[\begin{array}{ccc}
2 q_{0}^{2}+2 q_{1}^{2}-1 & 2\left(q_{1} q_{2}+q_{0} q_{3}\right) & 2\left(q_{1} q_{3}-q_{0} q_{2}\right) \\
2\left(q_{1} q_{2}-q_{0} q_{3}\right) & 2 q_{0}^{2}+2 q_{2}^{2}-1 & 2\left(q_{0} q_{1}+q_{2} q_{3}\right) \\
2\left(q_{0} q_{2}+q_{1} q_{3}\right) & 2\left(q_{2} q_{3}-q_{0} q_{1}\right) & 2 q_{0}^{2}+2 q_{3}^{2}-1
\end{array}\right]
$$

The 3-axis magnetometer gives the direction of the Earth's magnetic field in the body frame $B$ as follows:

$$
h=\left[\begin{array}{lll}
h_{x} & h_{y} & h_{z}
\end{array}\right]^{T}=M_{N}^{B}(q) m+\delta_{h}
$$

where

$$
m=\left[\begin{array}{lll}
m_{x} & 0 & m_{z}
\end{array}\right]^{T}=[\|m\| \cos (\theta) \quad 0 \quad\|m\| \sin (\theta)]^{T}
$$

$m$ represents the magnetic field vector measured in the navigation frame $N$. The theoretical model of the magnetic field nearest to reality considers a magnetic field vector with an inclination angle $\theta=60^{\circ}$ and a norm vector $\|m\|=0.5$ Gauss [20]. $\delta_{h} \in \mathfrak{R}^{3}$ is a vector of zero-mean white Gaussian noise and the rotation matrix $M_{N}^{B}(q)$ is as expressed in (7). The LMA is an estimator that uses the Earth's magnetic field $m$ and the gravity vector $g$ as vector observations to deduce the attitude $q_{m}$.

This algorithm is based on the followings steps:

1) Measure accelerometer and magnetometer readings $f$ and $h$, respectively.

2) Calculate $\hat{f}^{N}=\hat{q} \otimes f \otimes \overline{\hat{q}}$ and do the same for $\hat{h}^{N}$. Note that $\hat{f}^{N}$ and $\hat{h}^{N}$ represent the estimated acceleration and magnetic field values in the navigation frame $N$.

Note also that $\overline{\hat{q}}=\left[\begin{array}{llll}\hat{q}_{0} & -\hat{q}_{1} & -\hat{q}_{2} & -\hat{q}_{3}\end{array}\right]^{T}$ represents the quaternion inverse of $\hat{q}$.

3) Calculate the navigation error $\partial f=g-\hat{f}^{N}$ and do the same for $\partial h=m-\hat{h}^{N}$ in order to form $z=\left[\begin{array}{ll}\partial h & \partial f\end{array}\right]^{T}$.

4) Calculate the Jacobian matrix:

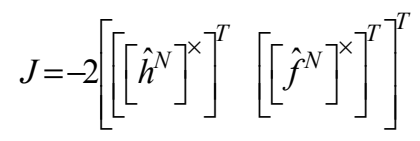

5) Calculate the pseudo-inverse $O^{*}=\left[J^{T} J+\lambda I_{3 \times 3}\right]^{-1} J^{T}$.

6) Calculate the quaternion error such as: $q_{e r}(t)=\alpha O^{*} z$. $\alpha$ is a smooth parameter chosen between 0 and 1 [11].
7) Calculate $q_{m}$ such as: $q_{m}(t)=\hat{q}(t) \otimes\left[\begin{array}{ll}1 & q_{e r}(t)\end{array}\right]^{T}$.

It is important to note that this algorithm provides a long term accuracy but still limited to the static or quasi-static situations (weak linear acceleration) [11]. Therefore, the values of $q_{m}$ are disturbed in dynamic situations (during high linear acceleration periods). In this paper, the introduction aim of the term $q_{m}$ is to correct the attitude errors caused by disturbances in the rate gyro measurements.

\section{Observer design}

The main emphasis of the proposed observer is based on the use of the multiplicative correction technique [15]:

$$
q^{\prime}=\delta q \otimes q
$$

Quaternion multiplication is used in (10) to correct and update the quaternion calculation process. $\delta q$ is the correction term which is a function of the measurement error.

To perform measurement update of the quaternion estimates, (10) can be transformed into the following form:

$$
\hat{q}^{+}=\delta \hat{q}^{+} \otimes \hat{q}^{-}
$$

where $\hat{q}^{+}$and $\hat{q}^{-}$represent the post and pre-update values of the quaternion estimates, respectively.

Finally, the Complementary Sliding Mode Observer (CSMO) can be designed by using (11) with the choice of a sliding manifold based on the error quaternion. The proposed observer is given as follows:

$$
\text { CSMO }:\left\{\frac{\partial \hat{q}}{\partial t}=\delta_{K_{1}} \otimes \delta_{K_{2}} \otimes \frac{1}{2}\left[\begin{array}{c}
-\hat{q}_{\text {vect }}^{T} \\
I_{3 \times 3} \hat{q}_{0}+\left[\hat{q}_{\text {vect }}^{\times}\right]
\end{array}\right] \omega_{g}\right.
$$

where $\hat{q}$ is the estimated quaternion at time $t$ and $\left[\hat{q}_{\text {vect }}^{\times}\right]$is defined as in (4). $\delta_{K_{1}}$ represents the switching correction term and $\delta_{K_{2}}$ is the linear correction term. In order to perform the quaternion multiplication, each correction term should be converted into a quaternion. This conversion is obtained using the forced normalization method given in [21].

$\delta_{K_{1}}$ and $\delta_{K_{2}}$ are computed such as follows:

$$
\delta_{K_{1}}=\frac{1}{\left\|\chi_{1}\right\|} \chi_{1} ; \quad \delta_{K_{2}}=\frac{1}{\left\|\chi_{2}\right\|} \chi_{2}
$$

where

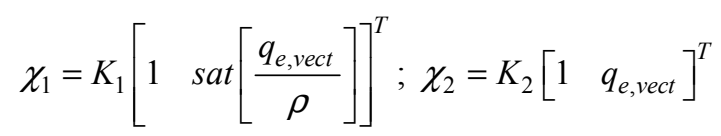

with

$$
K_{1}=\left[\begin{array}{cccc}
1 & 0 & 0 & 0 \\
0 & k_{1} & 0 & 0 \\
0 & 0 & k_{2} & 0 \\
0 & 0 & 0 & k_{3}
\end{array}\right] ; K_{2}=\left[\begin{array}{cccc}
1 & 0 & 0 & 0 \\
0 & k_{4} & 0 & 0 \\
0 & 0 & k_{5} & 0 \\
0 & 0 & 0 & k_{6}
\end{array}\right]
$$


Note that $q_{e, v e c t}$ is the imaginary vector of the error quaternion $q_{e}$. $q_{e}$ measures the discrepancy between the complementary estimated quaternion $\overline{\hat{q}}$ and the measured attitude $q_{m}$ ( $q_{m}$ is obtained from LMA, section III-B), that is:

$$
q_{e}=q_{m} \otimes \overline{\hat{q}}=\left[\begin{array}{ll}
q_{e 0} & q_{e, v e c t}
\end{array}\right]^{T}
$$

The scalar parts of $\chi_{1}$ and $\chi_{2}$ are chosen very close to unit since the incremental quaternion corresponds to a small angle rotation [11]. The saturation function sat is used to avoid the high frequency chattering behavior around the sliding surface:

$$
\operatorname{sat}\left(\frac{q_{e, v e c t}}{\rho}\right)= \begin{cases}1 & q_{e, v e c t} \leq \rho \\ \frac{q_{e, v e c t}}{\rho} & \left|q_{e, v e c t}\right| \leq|\rho| \\ -1 & q_{e, v e c t} \geq \rho\end{cases}
$$

The parameter $\rho$ is the sliding surface boundary layer. It determines the sliding behavior in the vicinity of $q_{e, v e c t}=0$. To preserve the unit quaternion norm, the estimated quaternion $\hat{q}$ in (12) must be normalized to avoid a divergence caused by round-off errors.

Normalization is obtained such as:

$$
\hat{q}_{\text {norm }}=\frac{\hat{q}}{\|\hat{q}\|}
$$

Fig. 2 illustrates the attitude estimation algorithm performed by the proposed nonlinear observer.

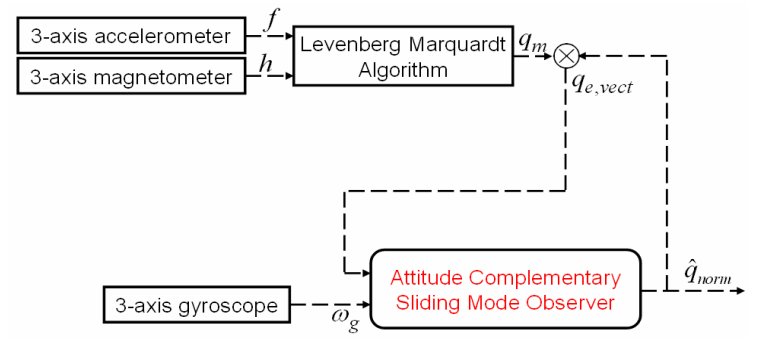

Fig.2. Scheme of the estimation algorithm

\section{Performance analysis of the designed observer}

A frequency analysis of inertial and magnetic sensors shows that signals coming from the accelerometermagnetometer pair and signals from the gyroscope have a complementary frequency spectrum [17]. Therefore, the resulting structure of the sliding mode observer (12) blends the low frequency region (low bandwidth) of the accelerometer and magnetometer data, where the attitude is typically more accurate, with the high frequency region (high bandwidth) of the gyroscope data, where the integration of the angular velocity yields better attitude estimates.

The proposed observer is derived from the complementary filtering theory [22]. It explores the sensor redundancy to reject measurement disturbances in complementary frequency regions without distorting the signal. If the measurements have complementary spectral characteristics, transfer functions may be chosen in such way as to minimize the estimation error [23]. The general requirement is that one of the transfer functions complements the sum of the others. Thus for $n$ measurements of a signal:

$$
1-T_{1}(s)-T_{2}(s)-\ldots-T_{n-1}(s)=T_{n}(s)
$$

where $s$ is the time integration Laplace operator.

To study the performance of the CSMO let us show the transform domain block diagram of the linearized quaternion observer (see Fig. 3). This diagram is obtained from Fig. 2.

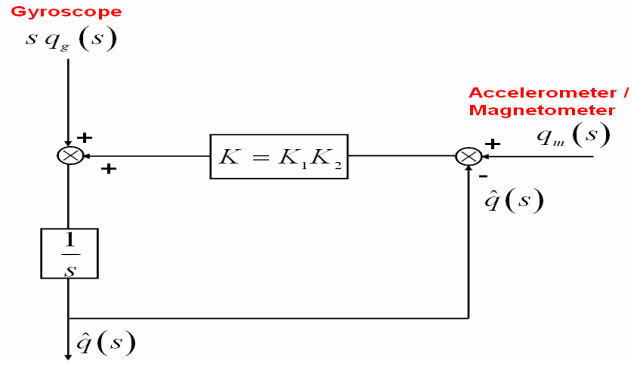

Fig. 3. Block diagram of the transform domain (Laplace) of the linearized quaternion estimation observer

Let the Laplace transform of the quaternion $q_{m}$ (obtained from accelerometer and magnetometer readings) be $q_{m}(s)$, while $q_{g}(s)$ is the quaternion obtained by integrating gyroscope signals and $s q_{g}(s)$ is the Laplace transform of $\partial q / \partial t$ in (3). Note that the accelerometer measures both gravitational and linear accelerations and the gyroscope suffers from bias. Therefore, $q_{m}(s)$ and $q_{g}(s)$ are both disturbed. From Fig. 3, the filter transfer function $F_{1}(s)$ based on accelerometer and magnetometer inputs is given by:

$$
F_{1}(s)=\frac{\hat{q}(s)}{q_{m}(s)}=\frac{K s^{-1}}{1+K s^{-1}}=\frac{K}{s+K}
$$

Note that (20) has the form of first-order low-pass filter which proves that the perturbation effects due to high frequency components of accelerometer signal (linear acceleration) are filtered from $q_{m}(s)$.

The gain $K$ can be written such as $K=K_{1} K_{2}$, where $K_{1}$ and $K_{2}$ are given in (15).

Similarly, from Fig. 3, the filter transfer function based on gyroscope input can be written such as:

$$
F_{2}(s)=\frac{\hat{q}(s)}{q_{g}(s)}=\frac{1}{1+K s^{-1}}=\frac{s}{s+K}
$$

Note that (21) has the form of first-order high-pass filter. The gyroscope measurements are high-pass filtered with respect to the output $\hat{q}(s)$. So, the perturbations due to low frequency components of gyroscope signal (the noises and biases) are filtered from $q_{g}(s)$.

Finally, (19) can be verified: 


$$
\frac{\hat{q}(s)}{q_{m}(s)}+\frac{\hat{q}(s)}{q_{g}(s)}=\frac{K}{s+K}+\frac{s}{s+K}=1
$$

Note that (22) confirms the complementary aspect of the CSMO. The global transfer function of the observer is:

$$
\hat{q}(s)=\left(\frac{K}{s+K}\right) q_{m}(s)+\left(\frac{s}{s+K}\right) q_{g}(s)
$$

One can conclude from (23) that CSMO blends a low-pass filtering on the signals from the accelerometer-magnetometer pair, and a high-pass filtering on the signals from the gyroscope.

\section{EXPERIMENTAL RESULTS}

At the time of the paper's writing, the final prototype (bilogger) is under conception. Experiments involving the king penguin will be carried out when the developed algorithm is implemented on the embedded prototype. Therefore, we chose to carry out the first preliminary experiment on another animal like a dog. The accuracy of the CSMO is evaluated during the free motion of the animal. An Inertial Measurement Unit was used, namely the MTi-G from Xsens Technologies [24]. It is important to note that the MTi-G serves as a tool for the evaluation of the CSMO efficiency and cannot be suitable for use in free-ranging animals due to its dependence on an energy source as well as its heavy weight. The MTi-G outputs data at a rate of $100 \mathrm{~Hz}$. The Xsens device is a miniature, light-weight, and 3D digital output sensor. It outputs 3D acceleration from a 3-axis accelerometer $\left( \pm 50 \mathrm{~m} / \mathrm{s}^{2}\right)$, 3D angular rate from a 3 -axis gyroscope $\left( \pm 300^{\circ} / \mathrm{s}\right)$, and 3D magnetic field data from a 3axis magnetometer $( \pm 750 \mathrm{mGauss})$ with built-in bias, sensitivity, and temperature compensation. Note that the MTi-G is a GPS enhanced Attitude and Heading Reference System (AHRS). The Xsens MT device can track the attitude in Euler angles and quaternion representations using an internal algorithm based on Xsens Kalman Filter (XKF) [24]. Before starting experiments, the MTi-G is attached to the back of the dog (see Fig. 4).
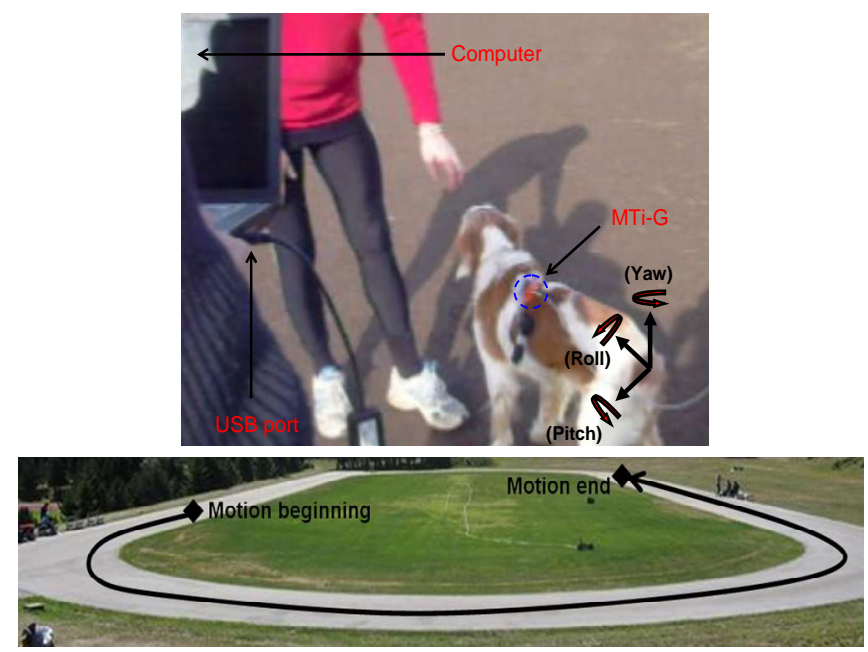

Fig. 4. The MTi-G attached to the back of the dog - Description of the dog motion
To represent more accurately the ongoing posture of the dog, some efforts are made to align the three axes of the body frame (the subject) with respect to the sensor frame so that the estimated orientation by the CSMO or by the MTi-G represents the real attitude of the dog. During the motion, the dog performed straight and curved trajectories in a football stadium (see Fig. 4). Its course is composed of natural gaits such as a walk, a trot, and a gallop. The recorded data from the MTi-G (3D-specific forces, 3D-angular rates, and 3DEarth's magnetic fields (see Fig. 5)) are used as inputs to the CSMO. For an initial period of time (between 0-18sec), the dog is at rest. Then, its accelerations represent only the gravity projection. When the animal starts the course (between $18 \mathrm{sec}-120 \mathrm{sec}$ ), the acceleration values increase and the variation range is between $-20 \mathrm{~m} / \mathrm{s}^{2}$ and $20 \mathrm{~m} / \mathrm{s}^{2}$.
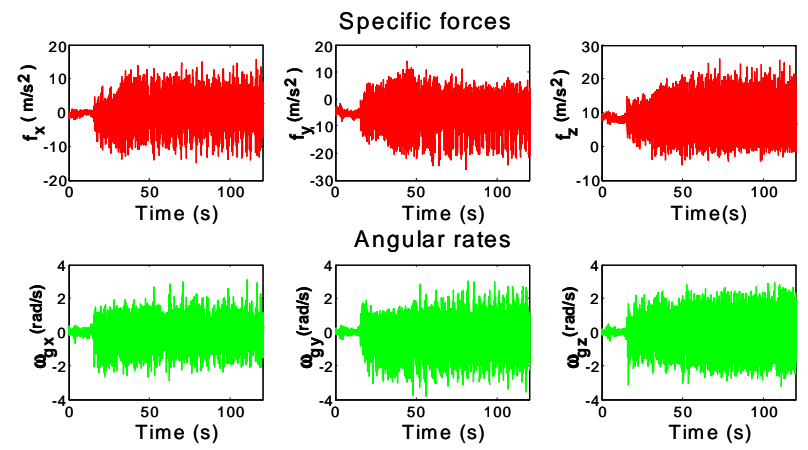

Earth's magnetic fields
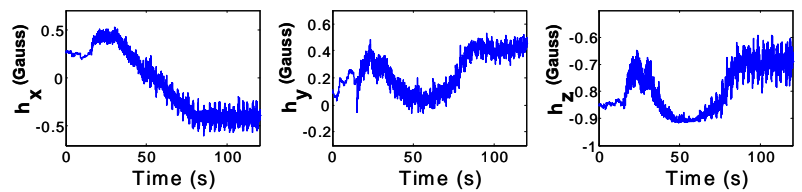

Fig. 5. Inertial and magnetic measurements from MTi-G

The quaternion estimation errors are shown in Fig. 6 to provide an overview of the overall performance of the nonlinear observer against a fusion method based only on accelerometer and magnetometer measurements. These errors are computed as the difference between quaternion estimates produced by the accelerometer/magnetometer fusion method (Acc/Mag) (Levenberg Marquardt Algorithm) (see Fig. 6(a)) [11] and MTi-G or by the CSMO and MTi-G (see Fig. 6(b)). Figure 6(a) shows large amounts of high errors because of the high frequency noise (motion accelerations) that affects the acceleration measurements. Conversely, Fig. 6(b) shows a small attitude estimation error during the motion of the animal. Hence, this figure illustrates the performance of the CSMO in estimating the attitude of the dog even in dynamic situations (gallop).

For this specific moving test, the performance of the CSMO is also compared with the fusion method (based on accelerometer and magnetometer measurements) by using the Root Mean Square Error (RMSE) criteria. Therefore, the RMSE differences on Euler angles between the CSMO and MTi-G outputs during the free movement experiments are computed. The same criterion is used to calculate the RMS differences between the accelerometer/magnetometer fusion method (Acc/Mag) and the MTi-G. The obtained results (see 
Table I) illustrate the performance level consistency of the CSMO in estimating the Euler angles with a small error.
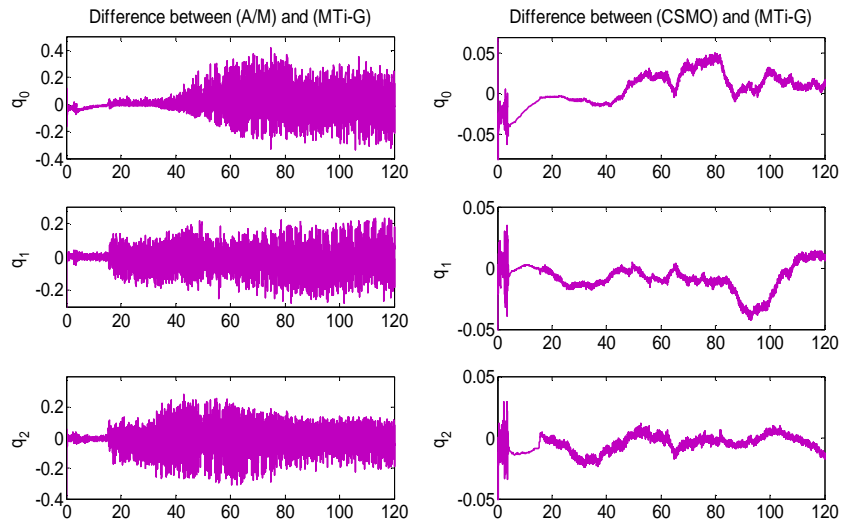

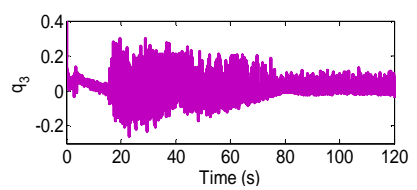

(a)

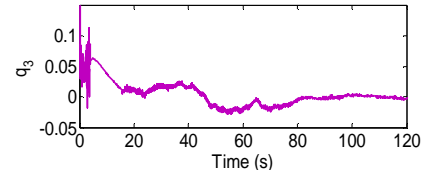

(b)
Fig. 6. (a) Differences between quaternion estimates produced by the fusion method (Acc/Mag) and MTi-G (b) Differences between quaternion estimates produced by the CSMO and MTi-G

TABLE I

RMSE DIFFERENCES ON EULER ANGLES

\begin{tabular}{ccc}
\hline & Fusion method (Acc/Mag) & CSMO \\
\hline RMSE $\left(^{\circ}\right)$ (roll) & 61.008 & 0.978 \\
RMSE $\left(^{\circ}\right.$ ) (pitch) & 50.335 & 1.333 \\
RMSE $\left({ }^{\circ}\right)$ (yaw) & 11.355 & 3.076 \\
\hline \hline
\end{tabular}

\section{CONCLUSION}

This paper focuses on the development of an alternative approach to recover the full attitude of a moving rigid body with application in Bio-logging area. Therefore, a Complementary Sliding Mode Observer (CSMO) is designed based on the complementary filtering theory and the multiplicative quaternion correction technique. By considering the rigid body kinematic model, the CSMO is proposed with a view to do a trade-off between a good short term precision given by rate gyros integration and a reliable long term accuracy provided by accelerometers and magnetometers measurements. The efficiency of the proposed approach is shown with experiments on a dog through sensor measurements provided by an Inertial Measurement Unit. The experimental results point out that the algorithm is able to track the accurate orientation under several motions. This algorithm will be used on the king penguin to assess its corresponding mechanical work.

\section{REFERENCES}

[1] S. P. N. Singh, and K. J. Waldron, "Attitude estimation for dynamic legged locomotion using range and inertial sensors," IEEE International Conference on Robotics and Automation, Barcelona, Spain, April 2005, pp. 1663-1668.

[2] R. Mahony, T. Hamel, and J. M. Pflimlin, "Nonlinear complementary filters on the special orthogonal group,", IEEE Transactions on Automatic Control, vol. 53, no. 5, pp. 1203-1218, June 2008.

[3] J. F. Vasconcelos, C. Silvestre, P. Oliveira, P. Batista, and B. Cardeira, "Discrete time-varying attitude complementary filter,"
IEEE American Control Conference, St. Louis, USA, June 2009, pp. 4056-4061.

[4] J. Thienel, and R.M. Sanner, "A coupled nonlinear spacecraft attitude controller and observer with an unknown constant gyro bias and gyro noise," IEEE Transactions on Automatic Control, vol. 48, no. 11, November 2003.

[5] P. Martin, and E. Salaun, "Invariant observers for attitude and heading estimation from low-cost inertial and magnetic sensors," IEEE Conference on Decision and Control, New Orleans, USA, December 2007, pp. 1039-1045.

[6] A. M. Sabatini, "Quaternion-based Extended Kalman Filter for determining orientation by inertial and magnetic sensing," IEEE Trans. on Biomedical Eng., vol. 53, no. 7, pp. 1346-1356, June 2006.

[7] V. Van Acht, E. Bongers, N. Lambert, and R. Verberne, "Miniature Wireless Inertial Sensor for Measuring Human Motions," $29^{\text {th }}$ Annual International Conference of the IEEE EMBS, Lyon, France, August 2007, pp. 6278-6281.

[8] H. Fourati, N. Manamanni, L. Afilal, and Y. Handrich, "Nonlinear attitude estimation based on fusion of inertial and magnetic sensors: Bio-logging application," IFAC International Conference on intelligent Control Systems and Signal Processing, Turkey, September 2009.

[9] H. Fourati, N. Manamanni, L. Afilal, and Y. Handrich, "A rigid body attitude estimation for Bio-logging application: A quaternion-based nonlinear filter approach," IEEE/RSJ International conference on Intelligent Robots and Systems IROS'09, St. Louis, USA, October 2009, pp. 558-563.

[10] C. Rutz, and G. C. Hays, "New frontiers in Bio-logging science," Biology letters, March 2009.

[11] G.H. Elkaim, E.B. Decker, G. Oliver, and B. Wright, "Marine Mammal Marker (MAMMARK) dead reckoning sensor for In-Situ environmental monitoring,", IEEE Position Location and Navigation Symposium, Monterey, USA, April 2006, pp. 976-987.

[12] M. P. Johnson, and P. L. Tyack, "A digital acoustic recording tag for measuring the response of wild marine mammals to sound," IEEE Journal of Oceanic Eng., vol. 28, no. 1, pp. 3-12, January 2003.

[13] R. P. Wilson, E. L. C. Shepard, and N. Liebsch, "Prying into the intimate details of animal lives: use of a daily diary on animals," Endangered Species Research, vol. 4, pp. 123-137, December 2007.

[14] M. S. Grewal, L. R. Weill, and A. P. Andrews, Global positioning systems, inertial navigation, and integration, John Wiley \& Sons, 2001.

[15] J.B. Kuipers, Quaternion and Rotation Sequences, Princeton, NJ: Princeton University Press, 1999.

[16] M.D. Shuster, "A survey of attitude representations", Journal of the Astronautical Science, vol. 41, no.4, pp. 493-517, 1993.

[17] R.G. Brown, and P.Y.C Hwang, Introduction to Random Signal and Applied Kalman Filtering, $3^{\text {rd }}$ Ed. New York: John Wiley, 1997.

[18] G. Wahba, "A least squares estimate of spacecraft attitude," SIAM, Review, vol. 7, no.3, pp. 409, 1965.

[19] H. Fourati, N. Manamanni, L. Afilal, and Y. Handrich, "Posture and body acceleration tracking by inertial and magnetic sensing: Application in behavioral analysis of free-ranging animals," Biomedical Signal Processing and Control, in press, June 2010, doi: 10.1016/j.bspc.2010.06.004.

[20] Astrosurf, January 2010. Available: http://www.astrosurf.com.

[21] J. Deutschmann, I. Bar-Itzhack, and K. Galal, "Quaternion normalization in spacecraft attitude determination," Proceedings of the AIAA Astrodynamics Conference, 1992, pp. 27-37.

[22] W. Higgins, "A comparison of complementary and Kalman filtering," IEEE Transactions on Aerospace and Electronic Systems, vol. 11, no. 3, pp. 321-325, 1975.

[23] H. Fourati, N. Manamanni, L. Afilal, and Y. Handrich, "Nonlinear Filtering Approach for the Attitude and Dynamic Body Acceleration Estimation Based on Inertial and Magnetic Sensors: Bio-logging Application," IEEE Sensors Journal, in press, July 2010, doi: 10.1109/JSEN.2010.2053353

[24] Xsens Technologies, (2010, February). Available: http://www.xsens.com 